\title{
Modeling low pressure collisional plasma sheath with space-charge effect
}

\author{
Subrata Roy ${ }^{\mathrm{a})}$ and B. P. Pandey \\ Computational Plasma Dynamics Laboratory, Mechanical Engineering, Kettering University, \\ Flint, Michigan 48532-4898 \\ Jonathan Poggie and Datta V. Gaitonde \\ Computational Sciences Branch, Air Vehicles Directorate, Air Force Research Laboratory, \\ Wright-Patterson AFB, Ohio 45433
}

(Received 4 October 2002; accepted 12 March 2003)

\begin{abstract}
The present work develops a computationally efficient one-dimensional subgrid embedded finite element formulation for plasma-sheath dynamics. The model incorporates space-charge effect throughout the whole plasma and the sheath region using multifluid equations. Secondary electron emission is not considered. A third-order temperature dependent polynomial is used to self-consistently calculate the rate of ionization in the plasma dynamic equations. The applications include $\mathrm{dc}$ and $\mathrm{rf}$ sheath inside a glow discharge tube where the noble gas is immobile, and a partially ionized plasma sheath inside an electric propulsion thruster channel in which the gas flows. The electron and ion number densities of the numerical solution decrease in the sheath region as expected. The ion velocity and electron temperature profiles also exhibit the expected behavior. The computed sheath potential compares well with the available experimental data. (C) 2003 American Institute of Physics. [DOI: 10.1063/1.1572491]
\end{abstract}

\section{INTRODUCTION}

Sheath formation at the plasma-wall interface separating the quasi-neutral plasma is essential to contain the wall potential. Accurate sheath modeling is of considerable interest to the effective design of ionized flow in wide ranging applications in plasma processing: in the ion cyclotron heating; in electric propulsion devices; in fusion plasmas; and in highspeed air vehicles. In the electric propulsion devices, the build up of sheath potential and its stability may severely affect the thruster efficiency. ${ }^{1}$ The wall interaction in a magnetically confined fusion plasma such as tokamak is important especially near the limiter and divertor. Understanding sheath is also vital in high-speed air vehicles due to recent disclosure of a Russian concept AJAX vehicle. ${ }^{2}$ Considerable reduction in the aerodynamic drag via plasma or high temperature gas injection from the stagnation region has been reported in weakly ionized gas. ${ }^{3,4}$ The drastic increase of the stand-off distance of a bow shock over a blunt body stands in stark contrast to all experimental data and calculations of nonequilibrium hypersonic flows in the absence of electromagnetic field. Experimental results over a Mach number ranging from 0.5 to 4.0 , document nearly $60 \%$ drag reduction under electromagnetic field. ${ }^{5}$ These effects can be further controlled by the existence of a plasma sheath near the leading surface of the air vehicle.

The present status of space propulsion and hypersonic flow research reflects a dearth of consistent numerical models to understand the effect of near wall plasma interaction. ${ }^{2}$ Despite some recent experimental and theoretical advances, we lack the proper model to adequately describe the un-

\footnotetext{
a) Author to whom correspondence should be addressed. Telephone: 810 762-9949. Electronic mail: sroy@kettering.edu
}

steady dynamics of critical regions of weakly ionized plasma in a self-consistent fashion. The anomalies are due to the choice of Bohm's criterion (which is appropriate for collisionless sheath) or its variations as the boundary condition for both plasma and collisional sheath using an inconsistent model. This paper describes the theoretical and numerical basis using multifluid equations without the influence of magnetic field where space-charge effect is included throughout the whole plasma and the sheath region.

The build-up of near wall potential due to different mobility of the constituents in an ionized gas is as old a problem as the discovery of plasma itself. ${ }^{6}$ The specific feature of the plasma sheath near an electrode is the formation of charged boundary layer. A stationary collisionless sheath exists only if the ion flow velocity satisfies the Bohm criteria at the plasma-sheath boundary, i.e., plasma drift speed must exceed the ambipolar ion sound speed (Bohm velocity). Though the particle mobility depends upon many factors, the principal factor in sheath plasma is the induced dc sheath potential which equates all fluxes, preserving in the process the quasineutrality of the bulk plasma. Thus, a sheath consists of a presheath which is of the order of mean free path of plasmaneutral interaction and where plasma maintains a spacecharge neutrality and a Debye sheath, which is of the order of Debye length $\lambda_{D}$ and where a large potential drop occurs. The ions are accelerated in the pre-sheath region so that they enter the sheath region with the minimum energy required for a stable sheath. The condition in the presheath-Debye sheath transition region depends on various parameters such as the ion flow velocity, the plasma temperature, the neutral density and so on.

Near the sheath region, elastic and inelastic collisions between ions and neutrals play an important role on the sheath dynamics. The one-dimensional analytical and nu- 
merical analyses of Valentini ${ }^{7-9}$ suggest that in a collisional plasma, the thickness of the sheath is not only substantially larger than the Debye length $\lambda_{D}$ but also, plasma drift velocity can be smaller than the Bohm velocity $V_{B}$. The edge of the region where electric field generates the sheath is shifted to an ion drift velocity larger than the Bohm velocity under the influence of collision. ${ }^{9}$ In the interval where the ion drift velocity is smaller than the critical velocity $V_{c}=V_{B} \sqrt{n_{i} / n_{e}}$, the effect of collision dominates the sheath formation. In the interval where ion drift velocity is larger than the critical velocity, both the collision and electric field play equally important role in the sheath formation. Therefore, defining the location of a sheath edge is difficult under collisional conditions. In fact no lower bound exists on the ion drift velocity in the collisional plasma ${ }^{9}$ and Debye length can be of the same order as the ion-neutral collisional mean free path, $\lambda_{\mathrm{mfp}} .{ }^{8}$ To describe the extension of the space charge sheath by collisions to ion velocities smaller than the Bohm velocity, Godyak and Sternberg ${ }^{10}$ introduce another characteristic velocity $V_{\mathrm{GS}} \approx V_{B}\left[1+\left(\pi \lambda_{D} / 2 \lambda_{i}\right)\right]^{-1 / 2}$ using a relatively plausible, but slightly arbitrary statement. Here $\lambda_{D}$ is the electron Debye length and $\lambda_{i}$ is the "effective" ion mean free path. Clearly, Bohm criterion is only sufficient but not a necessary condition for sheath formation. As shown in Ref. 9 the definitions of the sheath edge described in the literature ${ }^{8,10,11}$ are relatively arbitrary. To date, no sufficient experimental results exist to isolate the effect of collision.

Thus, it is worth asking how various parameters such as the interaction between the neutral and the plasma affect the formation of the sheath and how does, in the process, the ion acceleration in the channel get affected. The electron-neutral and ion-neutral collisions play key role in the momentum and energy transfer in a partially ionized plasma and their role on sheath formation is significant. On physical grounds one anticipates that the ion collision in the sheath may reduce the ion impact energy to the wall. Consequently, ion dynamics must encompass the entire range of collisionality. Valentini et $\mathrm{al}^{7}$ have used a steady state two-fluid model throughout the plasma up to the wall for analyzing current carrying sheath and presheath region.

The length scale disparity between the bulk plasma and the bounding sheath causes considerable numerical difficulties. A part of the literature on the plasma-sheath modeling suggests that sheath and the plasma region can be modeled separately and treat sheath as a boundary condition to the bulk plasma solution. ${ }^{10,11}$ However, for a time-dependent sheath, it is not clear how to properly match the sheath to the bulk plasma. ${ }^{12}$ Therefore, a combined plasma-sheath model development is appropriate where the space charge effect is incorporated for the entire region. Besides, without the participation of neutrals the effect of ionization and recombination cannot be studied satisfactorily. Recently, the threecomponent partially ionized plasma-sheath model with a constant electron temperature and neutral density have been investigated. ${ }^{9}$ In this paper, a three-fluid compressible plasma-sheath model is developed for a low pressure partially ionized plasma that includes the effect of ionization and recombination on the plasma dynamics in the presence of neutrals and isothermal ions. The neutral continuity equa- tion is included with a given, fixed uniform neutral velocity. The electron energy equation has also been solved for selfconsistent determination of ionization and recombination. While the space charge effect is applied throughout the whole plasma and the sheath region, the secondary electron emission is not considered.

The numerical simulation is based on two-momentum, single-temperature, three-fluid flow equations. The numerical details have been previously described. ${ }^{13,14}$ One-dimensional (1D) sub-grid embedded (SGM) finite elements ${ }^{15}$ has been utilized for convergence and stability of the steady state solution. The glow discharge tube is modeled with a working fluid of argon gas. For estimating the neutral-ion cross section and collision frequencies in the electric propulsion application, the neutral gas is assumed as inert, namely, xenon.

\section{MODEL DESCRIPTION}

We will present two different physical models for low pressure (up to few milliTorr) partially ionized gas. The first model is of dc and rf discharge tubes where the noble gas is immobile. The second is for electric propulsion applications where the gas flows.

\section{A. dc and rf discharge tubes (pressure $\sim 0.1$ Torr)}

Understanding the ionization process is of paramount importance to control the mechanism for practical highspeed design consideration. ${ }^{16}$ Significant studies of the discharge mechanism in gas lasers, high power switches, and plasma processing notwithstanding, accurate and selfconsistent numerical description for glow discharges are yet to be formulated. Most classical modeling efforts are focused on equilibrium approximation assuming the transport coefficients (drift velocity, diffusion coefficient, and ionization coefficient) to be functionals of the electric field. In the present study, physical model of the glow discharge is based on the fluid description of electrons and ions. ${ }^{17}$ Ion transport is described by the continuity and drift-diffusion momentum transport equations. The electron transport follows the first two moments of the Boltzmann equation, namely the continuity and the momentum transport. The potential field is determined by the Poisson equation. The following equations govern the system:

$$
\begin{aligned}
& \text { Transport: } \frac{\partial n_{\alpha}}{\partial t}+\frac{\partial\left(n_{\alpha} V_{\alpha}\right)}{\partial z}=n_{e} N k_{i}, \\
& \text { for } \alpha=e, i\left\{\begin{array}{l}
n_{i} V_{i}=n_{i} \mu_{i} E-D_{i} \frac{\partial n_{i}}{\partial z} \\
n_{e} V_{e}=-n_{e} \mu_{e} E-D_{e} \frac{\partial n_{e}}{\partial z}
\end{array}\right. \text {, }
\end{aligned}
$$

Potential: $\quad \varepsilon_{0} \nabla^{2} \varphi=-e\left(n_{i}-n_{e}\right)$.

For the dc sheath [see Fig. 2(a) below], the tube utilized argon gas. The electron mobility $\mu_{e}$ is given as: $p \mu_{e}=3$ $\times 10^{5} \mathrm{~cm}^{2} \mathrm{~V}^{-1} \mathrm{~s}^{-1}$ Torr, for $p=0.1$ Torr and the electron diffusion from Einstein relation is $D_{e}=\left(k_{B} T_{e} / e\right) \mu_{e}$. Corresponding ion diffusion $D_{i}=200 \mathrm{~cm}^{2} / \mathrm{s}$ at $300 \mathrm{~K}$, and the ion mobility is given as ${ }^{17}$ 


$$
\begin{aligned}
p \mu_{i}= & 10^{3}\left(1-2.22 \times 10^{-3} E / p\right) \mathrm{cm}^{2} \mathrm{~V}^{-1} \mathrm{~s}^{-1} \text { Torr, } \\
& \text { for } E / p \leqslant 60 \mathrm{~V} \mathrm{~cm}^{-1} \mathrm{Torr}^{-1}, \\
p \mu_{i}= & \frac{8.25 \times 10^{3}}{\sqrt{E / p}}\left(1-\frac{86.52}{(E / p)^{3 / 2}}\right) \mathrm{cm}^{2} \mathrm{~V}^{-1} \mathrm{~s}^{-1} \mathrm{Torr}, \\
& \text { for } E / p>60 \mathrm{~V} \mathrm{~cm}^{-1} \mathrm{Torr}^{-1} .
\end{aligned}
$$

For argon gas, the ionization rate is given by the modified Townsend equation

$$
\begin{aligned}
& N k_{i}=\mathrm{A} \exp \left(\frac{-\mathrm{B}}{(E / p)^{0.4}}\right) p \mu_{e} E \mathrm{~s}^{-1} \\
& \mathrm{~A}=34 \mathrm{~cm}^{-1} \text { and } \mathrm{B}=16[\mathrm{~V} /(\mathrm{cm} \text { Torr })]^{0.4} .
\end{aligned}
$$

The molecular ions and their role in glow discharge has been investigated extensively. ${ }^{18}$ In the present work, we shall neglect the interactions between charged particles and neutral atoms in the excited state. As a result, the effect of super elastic collisions on stepwise excitation or ionization is ignored. This assumption is valid for a low pressure ( 100-250 mTorr) discharge and low rf conditions. ${ }^{19}$

For the dc sheath model, the cathode is maintained at an imposed potential of $-150 \mathrm{~V}$ while the anode is at $0 \mathrm{~V}$. Vanishing ion and electron number densities are specified at the anode and cathode, respectively. The electron flux towards the anode is given as $n_{e}\left(k_{B} T_{e} / 2 \pi m_{e}\right)^{1 / 2}$, the corresponding ion flux is given by Eq. (1). The electron temperature is assumed isothermal at $1 \mathrm{eV}(\sim 11600 \mathrm{~K})$ while the ion is cold $(300 \mathrm{~K})$. All other boundary conditions are maintained at zero flux, i.e., homogeneous Neumann.

For the rf sheath model, the discharge tube is filled with argon gas at 0.1 Torr and electrode voltage is alternating with 13.56 MHz frequency and $V_{\text {rf }}=100 \mathrm{~V}$ [see Fig. 3(a) below]. Fluid model has traditionally been very useful for this pressure range.

\section{B. Low pressure $\left(\sim 10^{-3}\right.$ Torr $)$ propulsion applications}

We shall assume a partially ionized xenon gas. The plasma-neutral collision usually determines the kinetics of the motion. The electron-neutral collision frequency is given as $\nu_{\mathrm{en}}=n_{n}\left\langle\sigma_{\mathrm{en}} V_{e \mathrm{th}}\right\rangle$. Assuming typical electron thermal velocity $V_{e t h} \sim 10^{6} \mathrm{~m} \mathrm{~s}^{-1}$ (electron temperature of several $\mathrm{eV}$ ), with neutral atom density $n_{n} \sim 10^{19} \mathrm{~m}^{-3}$, and $\sigma_{\text {en }} \approx 27$ $\times 10^{-20} \mathrm{~m}^{2}$ for $\mathrm{Xe}$, we see that the electron-neutral collision frequency is $\nu_{\mathrm{en}} \sim 10^{6} \mathrm{~s}^{-1}$. The ion-neutral collision frequency $\nu_{\text {in }}$ is much smaller than electron-neutral collision frequency $\nu_{\mathrm{en}}$ as $V_{i \mathrm{th}} \sim 10^{3}-10^{4} \mathrm{~m} \mathrm{~s}^{-1}$.

The rate of ion production in a plasma is determined by the ionization frequency. The rate of ionization is given as

$$
S_{\text {ioniz }}=n_{e} n_{n}\left\langle V_{e t \mathrm{~h}} \sigma_{i}\left(V_{e t \mathrm{~h}}\right)\right\rangle=k_{i} n_{e} n_{n},
$$

where $\sigma_{i}$ is the total cross section of the process, $n_{e}$ is the electron number density, and process constant $k_{i}$ $=\left\langle\sigma_{i}\left(V_{e \mathrm{th}}\right) V_{e \mathrm{th}}\right\rangle$, where the averaging is done over the velocities of the electrons whose kinetic energy is sufficient for ionization. A general electron temperature dependent empirical formula can be fitted to the ionization process constant $k_{i}=\left[k_{i}^{0+}, k_{i}^{0++}, k_{i}^{1++}\right]$, where $0+, 0++$ and $1++$ corre- spond to the neutral to single and double and single to double ionization respectively. Note that for $1++, n_{n}$ becomes $n_{i}$ in Eq. (5). We shall use the following generalized process rate that is a sum of all three ionization rates ${ }^{13}$

$$
\begin{aligned}
k_{i}= & \left(-3.2087 \times 10^{-5} T_{e}^{3}-0.0022 T_{e}^{2}+0.7101 T_{e}-1.76\right) \\
& \times 10^{-14}
\end{aligned}
$$

where $T_{e}$ is given in $\mathrm{eV}$. The above estimate of ionization rate is based on the use of Darwin's cross section ${ }^{13}$ and Maxwellian distribution function. However, an alternate choice for the cross section and subsequent calculation of ionization rate may be Lotz's ${ }^{20,21}$ formula.

The dissociative recombination is the fastest mechanism of bulk recombination in a weakly ionized plasma. One notes that the dissociative recombination coefficient $\left(\sim 10^{-13} \mathrm{~m}^{3} / \mathrm{s}\right)$ decreases as $T_{e}^{-1 / 2}$ at room temperature and as $T_{e}^{-3 / 2}$ at higher temperature. ${ }^{22}$ In the high-density, lowpressure plasma, the concentration of molecular ion is too low for dissociative recombination to be important. Furthermore, since such a plasma is optically thick, the radiative escape factor is zero and then the only important recombination mechanism is three-body recombination. ${ }^{23}$ Thus, the probability of recombination is

$$
S_{\text {recom }}=n_{e} n_{i}\left\langle V_{e \mathrm{th}} \sigma_{e i}^{r}\left(V_{e \mathrm{th}}\right)\right\rangle=\alpha n_{e} n_{i},
$$

where recombination coefficient $\alpha$ can be approximated as ${ }^{23}$

$$
\alpha=1.09 \times 10^{-20} n_{e} T_{e}^{-9 / 2} \mathrm{~m}^{3} / \mathrm{s} .
$$

The cross section for charge exchange collisions for $\mathrm{Xe}-\mathrm{Xe}^{+}$is given by ${ }^{24}$

$$
\begin{aligned}
\sigma\left(\mathrm{Xe}-\mathrm{Xe}^{+}\right)= & {\left[a-b \log _{10}(\Delta u)\right]\left(E_{i} / E_{H}\right)^{-1.5} } \\
& \times 10^{-20} \mathrm{~m}^{2}
\end{aligned}
$$

where $a=181, b=21.2, E_{i}=12.13 \mathrm{eV}$-xenon ionization potential and $E_{H}=13.6 \mathrm{eV}$-hydrogen ionization potential.

For a relative velocity $\Delta u$ between 10 and 2 $\times 10^{3} \mathrm{~m} / \mathrm{s}$, the charge exchange cross section is between $10^{-20}$ to $10^{-19} \mathrm{~m}^{2}$. We now give the basic set of equations that describes the plasma-sheath dynamics under investigation.

The continuity equation for electrons and ions

$$
\frac{\partial n_{\alpha}}{\partial t}+\frac{\partial\left(n_{\alpha} V_{\alpha}\right)}{\partial z}=S_{\text {ioniz }}-S_{\text {recomb }} .
$$

Here $V_{\alpha}$ and $n_{\alpha}$ are the electron and ion velocities and number densities respectively for $\alpha=e$ and $i$, with $S_{\text {ioniz }}$ and $S_{\text {recomb }}$ given by Eqs. (5) and (7). The theoretical difference between the sheath model of Godyak and Sternberg ${ }^{10,11}$ and Nitschke and Graves, ${ }^{12}$ and the present paper are that (i) the latter approach incorporates neutral density and recombination and ionization effects and (ii) the latter includes spacecharge effect throughout the plasma and the sheath.

The neutral continuity equation is

$$
\frac{\partial n_{n}}{\partial t}+\frac{\partial\left(n_{n} V_{n}\right)}{\partial z}=S_{\text {recomb }}-k_{i}^{0+} n_{e} n_{n}-k_{i}^{0++} n_{e} n_{n} .
$$


The ion momentum equation is

$$
\begin{aligned}
\frac{\partial V_{i}}{\partial t}+V_{i} \frac{\partial V_{i}}{\partial z}= & -\frac{T_{i}}{m_{i} n_{i}} \frac{\partial n_{i}}{\partial z}+\left(\frac{Z e}{m_{i}}\right) E-\nu_{c}\left(V_{i}-V_{n}\right) \\
& +\left(\frac{m_{e}}{m_{i}}\right) \nu_{e i}\left(V_{e}-V_{i}\right)-0.5 \nu_{i n}\left(V_{i}-V_{n}\right) \\
& +\left(S_{\text {recomb }}-S_{\text {ioniz }}\right) \frac{V_{i}}{n_{i}},
\end{aligned}
$$

where the factor 0.5 before ion-neutral collision term in the right hand side comes from reduced mass $m_{i} m_{n} /\left(m_{i}+m_{n}\right)$ $\approx m_{i} / 2$. Here, $E$ is the electric field, and $\nu_{e i}$ is the electronion collision frequency, $\nu_{c}$ is the ion charge-exchange collision frequency, $e$ is the electron charge and $Z$ is the ionicity which in general, is a function of the electron temperature. In the present calculation, we have assumed $Z=1$. The electron momentum equation is given as

$$
\begin{aligned}
\frac{\partial V_{e}}{\partial t}+V_{e} \frac{\partial V_{e}}{\partial z}= & -\frac{1}{m_{e} n_{e}} \frac{\partial p_{e}}{\partial z}-\left(\frac{e}{m_{e}}\right) E-\nu_{e i}\left(V_{e}-V_{i}\right) \\
& -\nu_{e n}\left(V_{e}-V_{n}\right)+\left(S_{\text {recomb }}-S_{\text {ioniz }}\right) \frac{V_{e}}{n_{e}} .
\end{aligned}
$$

The electron energy equation is

$$
\begin{aligned}
\frac{3}{2}\left(\frac{\partial T_{e}}{\partial t}+V_{e} \frac{\partial T_{e}}{\partial z}\right) \\
=-T_{e} \frac{\partial V_{e}}{\partial z}+\nu_{e i}\left(V_{e}-V_{i}\right)^{2}+\nu_{e n}\left(V_{e}-V_{n}\right)^{2} \\
+\left(\frac{S_{\text {recomb }}-S_{\text {ioniz }}}{n_{e}}\right)\left(\frac{3}{2} T_{e}-E_{i}\right) .
\end{aligned}
$$

Above energy equation is rather simplified, as the thermal conduction term has not been considered. This may lead to a slight overestimate of the electron temperature in the acceleration region $(\geqslant 0.75)$ of the thruster. ${ }^{25}$

Finally, the Poisson's equation for potential is

$$
\varepsilon_{0} \nabla^{2} \varphi=-e\left(n_{i}-n_{e}\right) \text {. }
$$

We again note that the space-charge effects are calculated throughout the plasma. The contribution due to the exchange of random thermal energy has also been ignored and only the dominant contribution due to the exchange of mean flow energy between electrons and ions has been retained.

Before numerically solving above set of Eqs. (10)-(15), we normalize the physical variables. Temperature $T_{e}$ is normalized to first ionization potential of xenon, $T_{e}=E_{i}$ $=12.1 \mathrm{eV}$. All dependent variables can be normalized using

$$
\begin{aligned}
& V_{B}=\sqrt{\frac{T_{e}}{m_{i}}}=3 \times 10^{3} \mathrm{~m} / \mathrm{s} ; \\
& n_{\mathrm{ref}} \simeq 1.5 \times 10^{19} \mathrm{~m}^{-3} ; \\
& \nu_{\mathrm{ref}}=\sigma_{\mathrm{ref}} n_{\mathrm{ref}} V_{B}=6.7 \times 10^{4} \mathrm{~s}^{-1} ; \phi=\left(\frac{e \varphi}{T_{e}}\right),
\end{aligned}
$$

where $\sigma_{\text {ref }}=\sigma_{0} \sqrt{m_{i} / m_{e}}, \quad \sigma_{0} \cong 3.6 \times 10^{-20} \mathrm{~m}^{2}$ for Xe. The fundamental length scale $l_{0}$ can be defined in terms of characteristic Bohm velocity $V_{B}$ and collisional frequency, $l_{0}$ $=V_{B} / \nu_{\text {ref }}$. The time scale is $t_{0}=\nu_{\text {ref }}^{-1}$.

Specification of proper initial and boundary conditions are required in order to complete the formulation of the sheath problem. In a combined plasma-sheath model, boundary conditions are used to provide the coupling between plasma and sheath regions. ${ }^{12}$ The ions are generated within the plasma by electron collisions with the neutral gas that is flowing through the anode with the reference density $n_{\text {ref }}$. In the experiments, an upstream diffusion region with the ion motion towards the anode has been observed. ${ }^{26}$ The reverse ion flow is recombined at the anode, and remitted as neutral gas to the channel. Downstream in the channel, ions move away from the anode towards the acceleration zone. The ions motion in the diffusion region is similar to the ion motion between two concentric negatively charged electrodes, ${ }^{7}$ where both inner and outer cylinders are negatively biased and ion flow towards both the surfaces. We impose zero ion velocity at the upstream boundary $(x=0)$ near the anode, i.e., $V_{i}(0)=0$. The electron temperature at this upstream location is specified as $0.4 E_{i}(\sim 5 \mathrm{eV})$. The electron density vanishes at the cathode $(x=1)$. The neutral velocity is assumed to have a uniform value everywhere. The cathode is maintained at a negative potential of $-30 \mathrm{~V}$. Homogeneous Neumann (zero normal derivative, $\mathrm{d} / \mathrm{dx}=0$ ) condition is imposed on the remaining boundaries. A uniform initial condition is used at the beginning of the simulation.

\section{FINITE ELEMENT BASED SUBGRID MODELING}

The details of the finite element based numerical method utilized for this model have been documented earlier. ${ }^{15}$ It is based on a newly derived, nonlinear sub-grid embedded (SGM) nonhierarchical finite element basis for use in a discrete approximation of a weak statement algorithm for (1), (2) and (10)-(15). Recent developments in the area of subgrid scale resolution include hierarchical $h-p$ elements and inclusion of nodeless bubble functions. The current development is distinctly different from these approaches in employing strictly classical Lagrange basis methodology. This leads to the key theoretical observation that the SGM basis is applicable only to the dissipative flux vector term, ${ }^{15}$ hence the kinetic flux vector remains a "centered" construction for the parent strictly Galerkin weak statement. The key consistency and efficiency ingredient of the SGM element is use of static condensation to reduce element matrix rank to that of the linear basis for any embedded degree. This is in sharp contrast to traditional enriched basis FE/FD algorithms, since the SGM element does not allow matrix order escalation, hence computer resource demands.

The SGM element construction for a 1D model form of (1), (2) and (10)-(15) leads to a theoretical nonlinear monotonicity constraint via enforcement of a real eigenvalue spectrum for the algorithm stencil. Thereby, the theory predicts the optimal distribution of the SGM embedded parameter (set) on each element, hence the mesh. The generalization to nonuniform, $d$-dimensional discretizations leads to the po- 


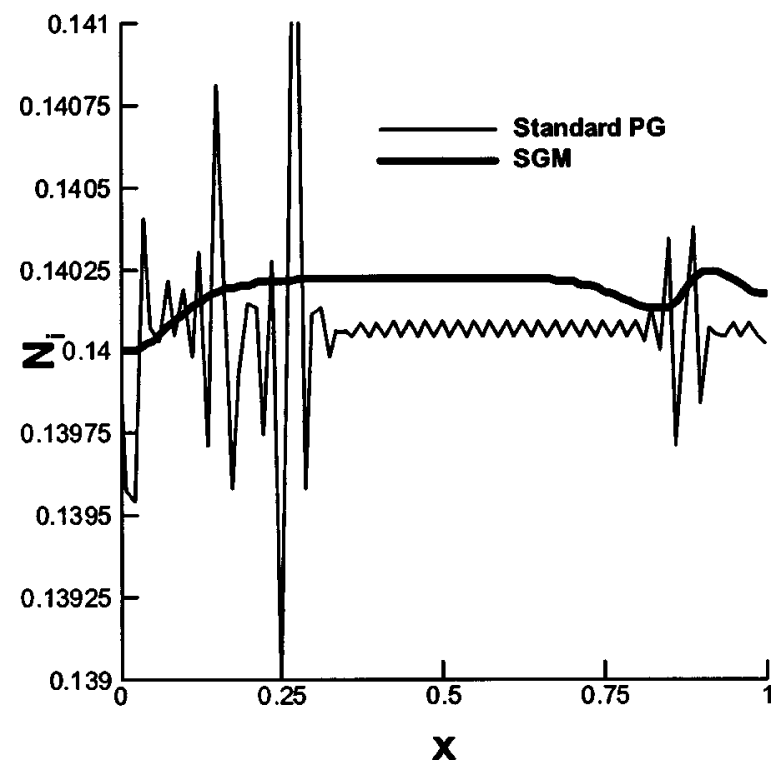

FIG. 1. Comparison between standard Petrov-Galerkin and subgrid embedded solutions for the ion number density distribution after $10^{-6} \mathrm{~s}$.

tential for attainment of nodally exact monotone solutions on arbitrary meshes. A comparison of standard Petrov-Galerkin and subgrid embedded method solution for the low-pressure electric propulsion plasma-sheath system (see Sec. IV B) is plotted in Fig. 1. Results on an 81 node mesh after $10^{-6} \mathrm{~s}$ shows the validity of SGM method in restraining solution oscillation. While the standard method completely diverges after $5 \times 10^{-6} \mathrm{~s}$, SGM results are robust and always nodewise monotone.

The choice of time step is dictated by the CourantFredrich-Levy condition. ${ }^{13}$ The code uses variable time steps until the transient features die down as the iteration converges to a steady state. Here, the convergence of a solution vector $\mathbf{U}$ on node $j$ is defined as the norm: $\| \mathbf{U}_{j}$ $-\mathbf{U}_{j-1}\|/\| \mathbf{U}_{j} \| \leqslant \epsilon$. The solution is declared convergent when the maximum residual for each of the state variable becomes smaller than a chosen convergence criterion of $\epsilon=10^{-4}$.

\section{RESULTS AND DISCUSSION}

As has been mentioned above, combined plasma-sheath dynamics is modeled by a 1D geometry. Equation sets (1), (2) and (10)-(15) are solved using SGM finite element method $^{15}$ over a computational domain $(x=z / L: 0,1)$ where $L$ is the characteristic length of the plasma-sheath region. The mesh is coarse and consists of 40 equal length 1D quadratic finite elements (i.e., 81 nodes) for all numerical results presented here.

\section{A. dc and rf discharge tube (pressure $\sim 1$ Torr)}

Figure 2 shows numerical simulation result for $1 \mathrm{~cm}$ long DC tube. A typical glow discharge tube light intensity, potential distribution, field strength, net space charge is documented in Ref. 23 (p. 451). The cathode is located at the left end while anode is at the right end. Numerical prediction for the potential field plotted in Fig. 2(a) using SGM finite elements demonstrates noticeable similarity with that in the
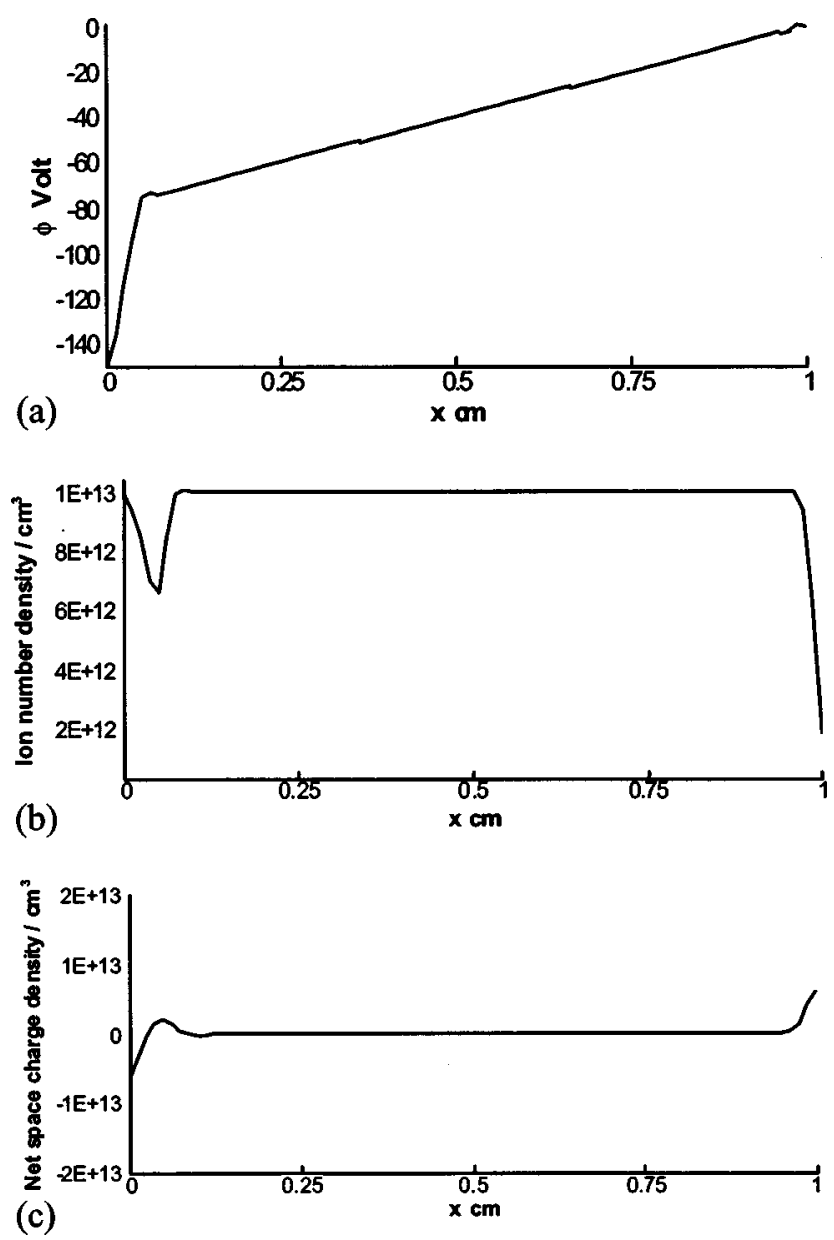

FIG. 2. DC discharge tube solution. (a) Calculated potential distribution. (b) Ion number density. (c) Net space charge $\left(n_{e}-n_{i}\right)$ inside the tube.

literature. ${ }^{27}$ The potential sharply rises from $-150 \mathrm{~V}$ to about $-75 \mathrm{~V}(\sim 50 \%)$ within the first $0.5 \mathrm{~mm}$ off the cathode surface indicating a drastic difference in ion and electron number densities near the cathode where the negative sheath is formed. At downstream of $0.5 \mathrm{~mm}$, the potential slightly drops before steadily rising towards the anode where it again changes the slope at about $9.7 \mathrm{~mm}$ indicating a small peak at the anode which is kept at $0 \mathrm{~V}$. The ion number density $\left(n_{i}\right)$ and net space-charge density distribution are shown in Figs. 2(b) and 2(c), respectively.

The charge oscillation near the cathode is similar to that of the representative distribution in the literature. ${ }^{27}$ The net space charge changes from negative to positive and then drops to zero within the first $1 \mathrm{~mm}$ near cathode. Most of the tube $(\sim 85 \%)$ shows a vanishing space charge, i.e., quasineutrality. Near the anode the space charge sharply rises to a positive peak. The calculated current for this case is about $0.1 \mathrm{~mA}$. For the cathode area of $2.4 \mathrm{~cm}^{2}(1 \mathrm{~cm}$ long and 2.4 $\mathrm{cm}$ wide), the current density is $42 \mu \mathrm{A} / \mathrm{cm}^{2}$.

Figure 3(a) shows the rf glow discharge tube schematic. Corresponding rf sheath solution plasma number density prediction $\left(n_{i} \times 10^{-9} \mathrm{~cm}^{-3}\right)$ is plotted in Fig. 3(b). The numerical prediction compares within $22 \%$ of the experimental data ${ }^{19}$ shown as filled triangles and plots similar trend as that of the reported numerical result ${ }^{17}$ in filled squares. 
RF Discharge Tube
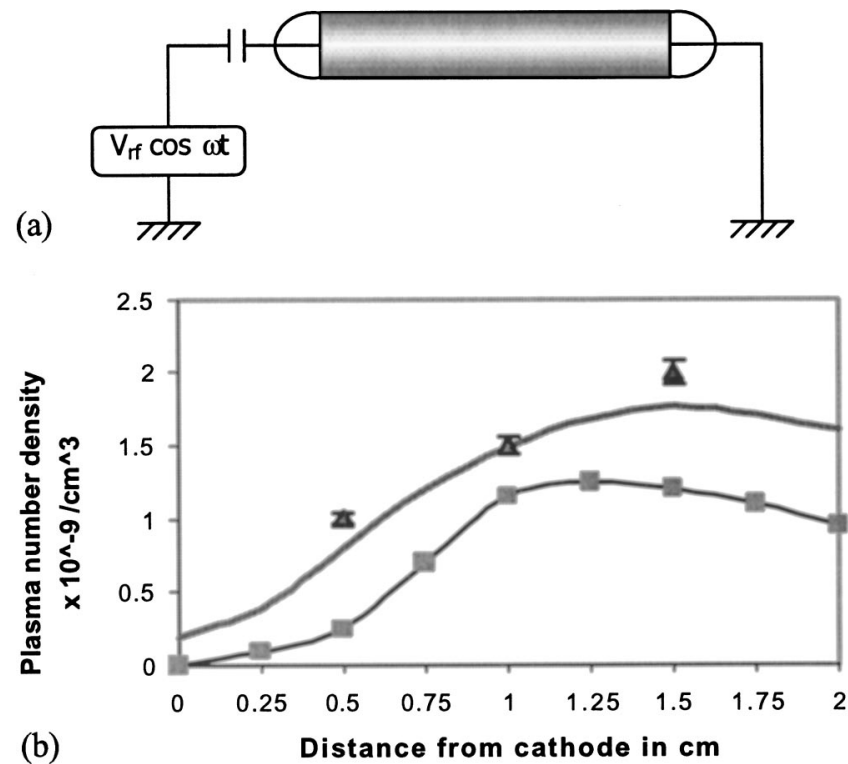

FIG. 3. Finite element solution (bold line) is compared with experimental data (Ref. 19) (filled triangles) and reported numerical result (Ref. 17) (filled squares). (a) rf discharge tube. (b) Plasma number density.
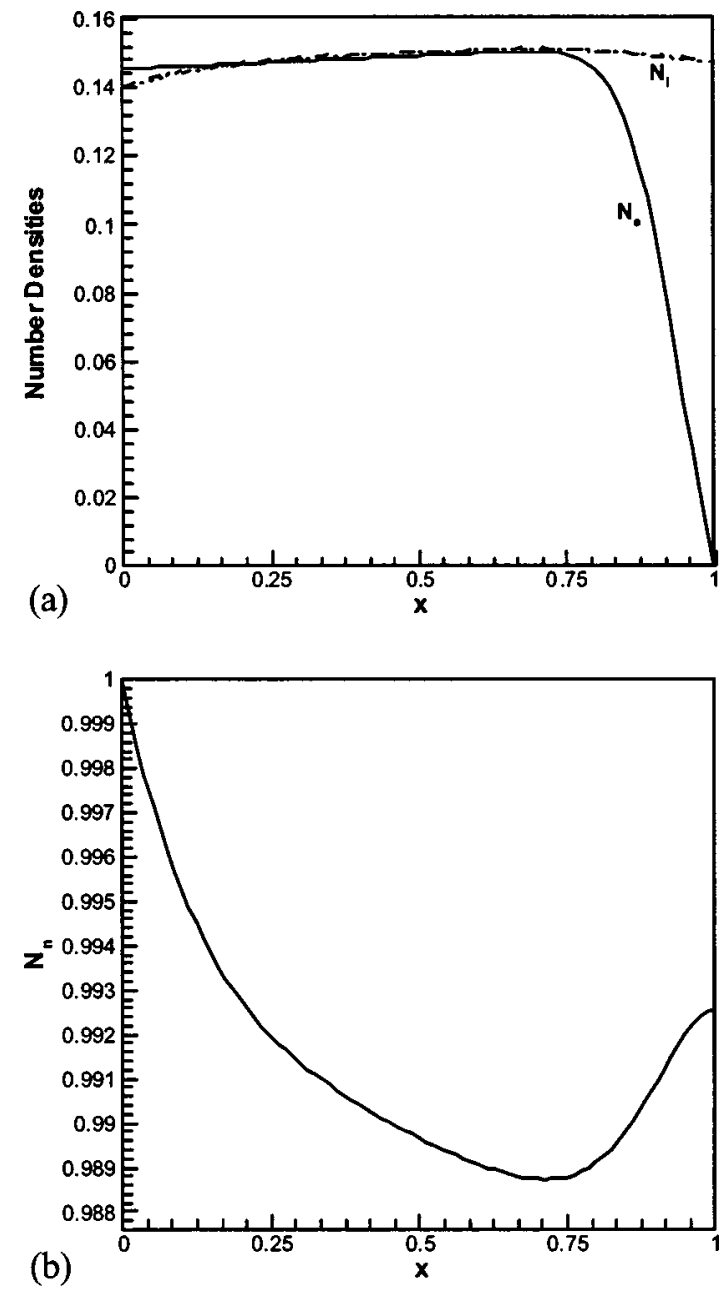

FIG. 4. (a) Ion and electron number densities normalized by the reference density $n_{\text {ref }}$. (b) Neutral number density distribution normalized by the reference density $n_{\text {ref }}$.

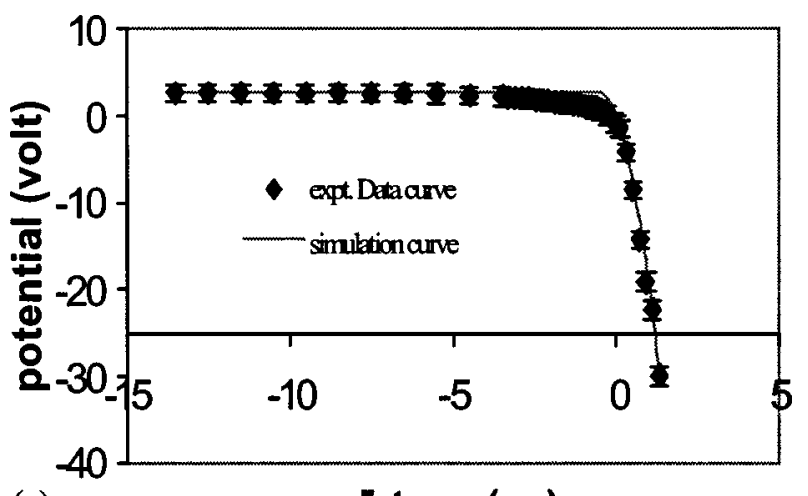

(a)

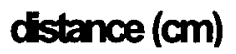

(b)

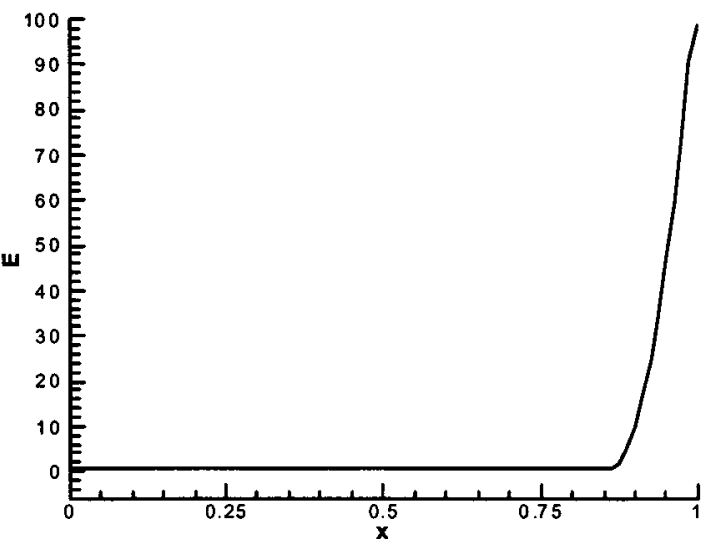

FIG. 5. (a) Plasma potential distribution compared with experimental data (Ref. 29) and (b) corresponding normalized electric field distribution.

\section{B. Low pressure $\left(\sim 10^{-3}\right.$ Torr $)$ propulsion applications}

The location of cathode and anode in this case are reverse of that of Figs. 2 and 3 (Sec. IV A). The electron, ion and neutral densities [Figs. 4(a) and 4(b)], and plasma potential and electric field [Figs. 5(a) and 5(b)] show the expected behavior. ${ }^{28,29}$ Far from the electrodes, a quasi-neutral plasma exists. In front of the electrode, a sheath is formed, more distinctly at the cathode than at the anode. A numerical tolerance $\left(\lambda_{D} / l_{0} \sim 0.05\right)$ has been utilized to quench the Poisson equation for potential [Eq. (15)]. Thus the electric field is zero at $x=0$ [Fig. 5(b)] in spite of a small variation of number densities [Fig. 4(a)]. The small decrease of $n_{n}$ is caused by the ionization process [Fig. 6(b)]. The reason for small increase of $n_{n}$ near the cathode may be due to the numerical errors and needs further investigation. Figures 6(a) and 6(b) plot the ionization and recombination rates and their combined effect. As the temperature increases, the ionization rate $k_{i}$ increases while the recombination rate $\alpha$ quickly tapers off and becomes very small near the wall as shown in Fig. 6(a). The combined effect of ionization and recombination, i.e., $S_{\text {ioniz }}-S_{\text {recom }}$ is documented in Fig. 6(b).

In Fig. 7(a), the ions are accelerated toward the negatively charged wall and reach the characteristic velocity $V_{\mathrm{GS}}$ given by Godyak and Sternberg ${ }^{11}$ at the presheath-sheath boundary. The ion velocity keeps increasing inside the sheath exceeding Bohm velocity and it finally saturates at the wall. 

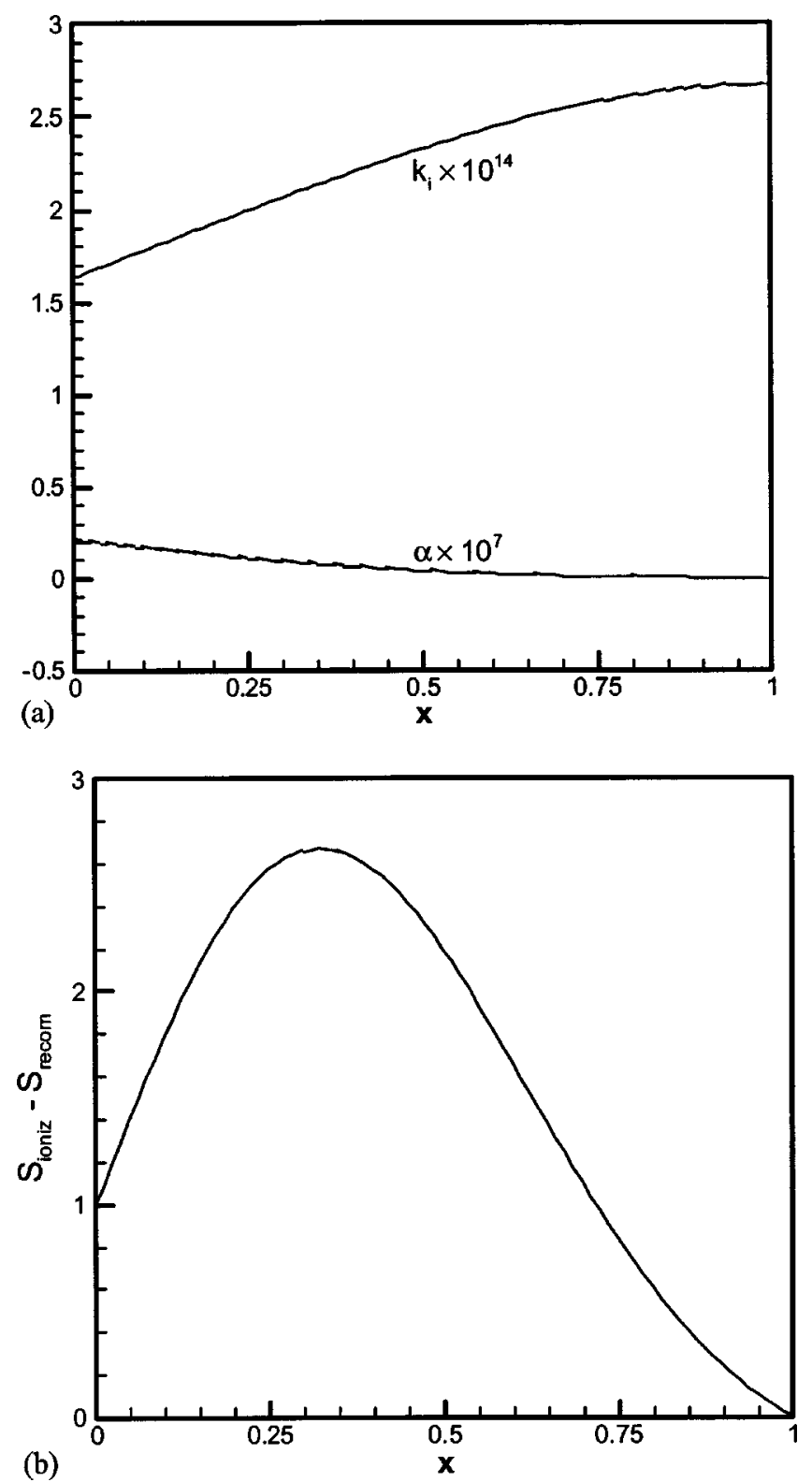

FIG. 6. (a) Ionization and recombination rates as a function of location $x$. (b) Source term $\left(S_{\text {ioniz }}-S_{\text {recom }}\right)$ in Eq. (10) normalized by the source value at $x=0$.

The characteristic velocities of Bohm $\left(V_{B}\right)$, Sternberg and Godyak $\left(V_{\mathrm{GS}}\right)$, and Valentini $\left(V_{C}\right)$ are identified in the figure for reference. We have noted above that the reduction of ion density inside the sheath can be correlated with the increase in the ion velocity. However, the process remains unclear in the bulk plasma region where also, ion velocity keeps increasing. The plasma is not in equilibrium and thus, above interpretation requires some caution as other processes like charge exchange collision, multiple ionization along with the elastic and inelastic processes are taking place simultaneously. Thus, ion velocity can increase, e.g., at the expense of, neutral velocity. This can be seen clearly if we switch off all other terms in ion momentum equation (12) except ionneutral collision. We see that constant neutral velocity will increase the ion velocity by $\sim\left(z \nu_{i n} V_{n}\right)^{1 / 2}$ which explains the initial rise in Fig. 7(a).
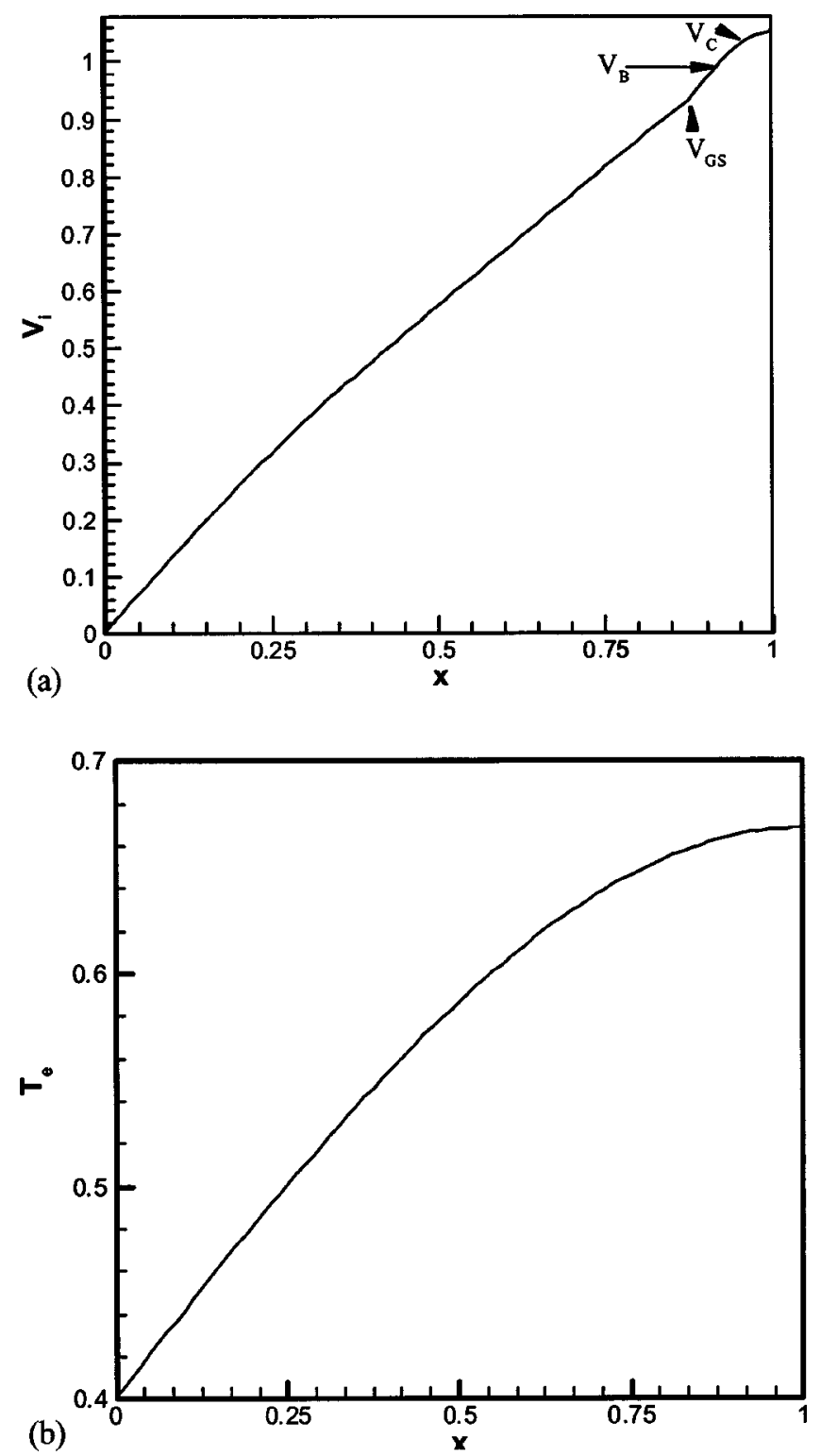

FIG. 7. (a) Normalized ion velocity profile shows the characteristic velocity of Sternberg and Godyak $\left(V_{\mathrm{GS}}\right)$, Bohm $\left(V_{B}\right)$, and Valentini $\left(V_{C}\right)$. (b) Electron temperature distribution normalized by the reference temperature.

The electron temperature profile is shown in Fig. 7(b). We note that the spatial evolution of density (Fig. 4) is correlated with the temperature evolution [Fig. 7(b)]. In fact this correlation could be anticipated on the physical ground. The increase in the electron temperature is not rapid. Further, close to the wall, the temperature profile saturates. The effect of temperature on the sheath is only indirect in the present model and requires further investigation.

\section{CONCLUSIONS}

In this paper, a subgrid embedded finite element based 1D formulation of plasma-sheath is given for an ionized gas using the three-component fluid equation. The numerical method is stable and nodewise monotone. This suggests that the subgrid embedded finite element method is a useful tool to solve multi-fluid equations in a low pressure plasma including the space charge. The applications include dc and rf 
sheath inside a glow discharge tube and a partially ionized plasma sheath inside an electric propulsion thruster channel. Based on the experimental data for multiple ionization of xenon gas, a third-order polynomial has been used in electron temperature as a fit to these processes.

The $\mathrm{dc}$ and $\mathrm{rf}$ sheath models compare reasonably well with available data in the literature. For the very low pressure thruster, the electron and ion number density profiles near the sheath show their usual distribution. The ion velocity keeps increasing in the bulk plasma and crosses the characteristic velocity given by Godyak and Sternberg near the pre-sheath. The sheath potential compares well with available experimental data. The model needs to be generalized in the near future to address the issues of secondary emission and magnetic field. More focused study is needed to understand the anomalous behavior of the neutral density near the wall.

\section{ACKNOWLEDGMENTS}

Part of this paper was written during the first author's tenure as National Research Council fellow at the Air Force Research Laboratory in Dayton, Ohio between May and July of 2002. S.R. and B.P. are also grateful for the support of NASA Grant Nos. NAG3-2520 and NAG3-2638.

${ }^{1}$ A. I. Morozov and V. V. Savelyev, in Reviews of Plasma Physics, edited by B. B. Kadomtsev and V. D. Shafranov (Consultants Bureau, New York, 2000), Vol. 21, p. 261.

${ }^{2}$ E. P. Gurijanov and P. T. Harsha, 7th International Space Planes and Hypersonic Systems and Technologies Conference, Norfolk, VA, 8-22 November 1996 (AIAA, Washington, DC, 1996) 96-4609.
${ }^{3}$ J. S. Shang, Prog. Aerosp. Sci. 37, 1 (2001).

${ }^{4}$ R. W. Ziemer, Amer. Rocket Soc. J. 29, 642 (1959).

${ }^{5}$ Y. C. Ganiev, V. P. Gordeev, A. V. Krasilnikov, V. I. Lagutin, V. N. Otmennikov, and A. V. Panasenko, J. Thermophys. Heat Transfer 14, 10 (2000).

${ }^{6}$ I. Langmuir and L. Tonk, Phys. Rev. 34, 874 (1929).

${ }^{7}$ H.-B. Valentini, E. Glauche, and D. Wolff, Plasma Sources Sci. Technol. 4, 353 (1995).

${ }^{8}$ H.-B. Valentini, Phys. Plasmas 3, 1459 (1996).

${ }^{9}$ H.-B. Valentini, Phys. Source Sci. Tech. 9, 1574 (2000).

${ }^{10}$ N. Sternberg and V. A. Godyak, Physica D 97, 498 (1996).

${ }^{11}$ V. A. Godyak and N. Sternberg, Phys. Rev. A 42, 2299 (1990).

${ }^{12}$ T. E. Nitschke and D. B. Graves, IEEE Trans. Plasma Sci. 23, 717 (1995).

${ }^{13}$ S. Roy and B. P. Pandey, Phys. Plasmas 9, 4052 (2002).

${ }^{14}$ S. Roy and B. P. Pandey, J. Plasma Phys. 68, 305 (2003).

${ }^{15}$ S. Roy and A. J. Baker, Numer. Heat Transfer, Part B 33, 5 (1998).

${ }^{16}$ T. C. Corke, E. J. Jumper, M. L. Post, D. Orlov, and T. E. McLaughlin, AIAA-2002-0350 (AIAA, Washington, DC, 2002).

${ }^{17}$ J. F. Boeuf and L. C. Pitchford, Phys. Rev. E 51, 1376 (1995).

${ }^{18}$ K. Kutasi, P. Hartmann, and Z. Donko, J. Phys. D 34, 3368 (2001).

${ }^{19}$ L. J. Overzet and M. B. Hopkins, J. Appl. Phys. 74, 4323 (1993).

${ }^{20}$ W. Lotz, Astrophys. J., Suppl. 14, 207 (1967).

${ }^{21}$ W. Lotz, Z. Phys. 216, 241 (1968).

${ }^{22}$ Y. P. Raizer, Gas Discharge Physics (Springer-Verlag, New York, 1991).

${ }^{23}$ M. Mitchner and C. H. Kruger, Partially Ionized Gases (WileyInterscience, New York, 1973).

${ }^{24}$ S. Pullins, Y. Chiu, D. Levandier, and R. Dressler, 38th Aerospace Sciences Meeting and Exhibit, Reno, NV, 2000 (AIAA, Washington, DC, 2000), AIAA-2000-0603.

${ }^{25}$ E. Ahedo, J. Gallardo, and M. Martinez-Sanchez, Phys. Plasmas 9, 4061 (2002).

${ }^{26}$ A. Bishaev and V. Kim, Sov. Phys. Tech. Phys. 23, 1055 (1978).

${ }^{27}$ M. A. Libermann and A. J. Lichtenberg, Principles of Plasma Discharges and Materials Processing (Wiley-Interscience, New York, 1994).

${ }^{28}$ F. F. Chen, Introduction to Plasma Physics (Plenum, New York, 1974).

${ }^{29}$ L. Oksuz and N. Hershkowitz, Phys. Rev. Lett. 89, 145001 (2002). 